\title{
A STUDY ON REGULARIZATION FUNCTIONS AND REGULATION PARAMETERS IN IMAGE RESTORATION
}

\author{
A.K. Kumaresh \\ Associate Professor Department of Computer Science \\ The M.D.T. Hindu College Tirunelveli, Tamilnadu, India
}

\begin{abstract}
The aim of this paper is to apply the regularization functions namely $T V$ norm, $l_{1}$ norm and $l_{0}$ norm and regularization parameters with these norms in image restoration. This class of problems results from combining a linear observation model with a non-quadratic regularizer. Improved Iterative Shrinkage Thresholding algorithm (IISTA) and Iterative Shrinkage Thresholding algorithm (ISTA) are employed for comparison. These algorithms are performed through a recursive application of two simple procedures such as linear filtering and soft thresholding.
\end{abstract}

Key words: ISTA, IISTA, image restoration, inverse problems, $1_{0}$ norm, $1_{1}$ norm, $1_{2}$ data fidelity term, regularization function, total variation.

Cite this Article: A.K. Kumaresh, A Study on Regularization Functions and Regulation Parameters in Image Restoration, International Journal of Computer Engineering and Technology 10(3), 2019, pp. 102-109.

http://iaeme.com/Home/issue/IJCET?Volume=10\&Issue=3

\section{INTRODUCTION}

Inverse problems abound in many application areas of image processing, remote sensing, radar imaging, tomographic imaging, microscopic imaging, astronomic imaging, digital photography [1][2][3][12]. Image restoration is one of the earliest and most classical linear inverse problems in imaging dating back to the 1960s [1].In a inverse problem, the goal is to estimate an unknown original image $\mathbf{x}$ from a noisy observation $\mathbf{y}$, produced by an operator $\mathbf{K}$ applied to $\mathbf{x}$, when $\mathbf{K}$ is a linear. Many applications to linear inverse problems LIPs define a solution $\mathrm{x}$ a restored image as a minimizer of a convex objective function $f: x \rightarrow \mathbb{R}=$ $[-\infty,+\infty]$ given by

$$
f(x)=1 / 2\|y-K x\|^{2}+\lambda \phi(x)
$$

where $\mathrm{K}: \mathrm{X} \rightarrow \mathrm{Y}$ is the linear direct operator, $\mathrm{X}$ and $\mathrm{Y}$ are real Hilbert spaces (both with norm denoted as $\|\cdot\|), \phi: X \rightarrow R$ is a function, $\lambda \in[0,+\infty]$ is a parameter.

In a regularization framework minimizing $\mathrm{f}$ is seen as a way of overcoming the illconditioned or singular nature of $\mathrm{K}$, which precludes inverting it. In this context $\phi$ is called regularizer and $\lambda$ is called regularization parameter [5]. 
In a finite dimensional Bayesian setting, the reasoning behind (1) is as follows: Assume that $\mathrm{y}=\mathrm{kx}+\mathrm{w}$, where $\mathrm{w}$ is a sample of a white zero-mean Gaussian random vector/field of variance $\sigma^{2}$, let $\mathrm{p}(\mathrm{x})$ be the adopted prior, thus the logarithm of a posteriori density is $\log p(x \mid y)=-f(x)$ upto a constant with $\lambda=\sigma^{2}$ and $\phi(x)=-\log p(x)$ : maximum posteriori (MAP) estimate are thus minimizer of $\mathrm{f}$. Despite the possible interpretation of (1)

$\phi$ simply referred as the regularize in equation (1). The intuitive meaning of $\mathrm{f}$ is simple: minimizing it corresponds to looking for a compromise between the lack of fitness of a candidate estimate $\mathrm{X}$ to the observed data, measured by $\|y-K x\|^{2}$ and its degree of undesirability given by $\phi(x)$. The regularization parameter $\lambda$ controls the relative weight of the two terms. Examples of total variation (TV) regularization [8][13] and wavelet based regularization [14][15].The non-differentiable nature of $\mathrm{f}$ together with the huge dimension of its argument for example $512 \times 512$ image $X=\mathrm{R}^{262144}$, place its minimization beyond the reach of standard off-the-shelf optimization methods.

This paper strictly concerned with algorithms for minimization [1] and discusses different choices of $\lambda$ and $\emptyset$. The rest of the paper is organized as follows. Section 2 discusses preliminaries. Section 3 explains proposed work. Section 4 analyses the experimental results. Section 5 concludes the paper.

\section{PRELIMINARIES}

\subsection{General Restoration Model}

In general, the image restoration problems have the form

$$
\min _{x \in R} n \varphi(x)=f(x)+\tau c(x)
$$

$\mathrm{f}: R^{n} \rightarrow R$ is smooth and convex data fidelity term, usually

$$
f(x)=\frac{1}{2}\|A x-y\|_{2}^{2}
$$

$\mathrm{C}: R^{n} \rightarrow R$ is a regularization or penalty function, typically convex often non-differentiable.

If $\mathrm{A}=\mathrm{I}$, it has a denoising problem.

If $\mathrm{c}$ is a proper and convex $\varphi$ is strictly convex, there is a unique minimizer. Thus the shrinkage thresholding function is

$$
\Psi_{\lambda}=\arg _{z}^{\min } \frac{1}{2}\|Z-u\|_{2}^{2}+\lambda c(z)
$$

is a well defined Moreau-proximal mapping.

\section{PROPOSED WORK}

\subsection{Regularization Functions}

Three types of regularization functions $l_{0}$ norm, $l_{1}$ norm and TV norm are studied with respect to restoration. Iterative shrinkage thresholding algorithm (ISTA) [9] and Improved Iterative shrinkage thresholding (IISTA) [10] is used to measure the performance.

If $c(z)=\|z\|_{0}, l_{0}$ norm then $\Psi_{\lambda}=\operatorname{hard}(z, \lambda)$ where $\operatorname{hard}(z, \lambda)=x^{*}(\operatorname{abs}(\mathrm{x})>=\operatorname{sqrt}(2 * \lambda))$. 
If $c(z)=\|z\|_{1}, l_{1}$ norm then $\Psi_{\lambda}(z)=\operatorname{soft}(z, \lambda)$ where

$\operatorname{soft}(z, \lambda)=\operatorname{sign}^{*}(|z|-\lambda)_{+}$and

$$
(a)_{+}= \begin{cases}0 & \text { if } a<0 \\ a & \text { if } a \geq 0\end{cases}
$$

Note that both functions are component wise application

If $\mathrm{c}(\mathrm{z})=\mathrm{TV}(\mathrm{z})$, total variation function then equation becomes

$$
\Psi_{\lambda}(z)=\arg _{z}^{\min } \frac{1}{2}\|A z-y\|_{2}^{2}+\tau T V(z)
$$

Where $T V(z)=\sqrt{\left(\Delta_{i}^{h} z\right)^{2}+\left(\Delta_{i}^{v} z\right)^{2}} \quad$ where $\Delta_{i}^{h}(\mathrm{z})$ and $\Delta_{i}^{v}(\mathrm{z})$ are linear operators corresponding to horizontal and vertical first order differences at point $\mathrm{i}$ respectively i.e, $\Delta_{i}^{h} z=z_{i}-z_{j}$ where $\mathrm{j}$ is the first order neighbour to the left of $\mathrm{i}$, and $\Delta_{i}^{v} z=z_{i}-z_{k}$ where $\mathrm{k}$ is the first order neighbour above $i$. This equation is isotropic and not differentiable.

$\tau$ is a regularization parameter in equation (5) are analyzed for three types regularization functions.

\section{EXPERIMENTAL RESULTS}

The Experiments are carried with MATLAB R2010a. The role of regularization functions and regularization parameters are studied using ISTA and IISTA algorithms under blur image of size $4 \times 4$ and $9 \times 9$. The increase of blur size shows that increase of iterations for restoration

Tables 1-4 gives result of regularization function role with number of iterations. The study of regularization parameter $\boldsymbol{\tau}$ with ISNR, objective function and regularizations is consolidated in Tables 5-8.

Table 1 ITERATIONS vs. ISNR using ISTA Blur size 9x9

\begin{tabular}{|c|c|c|c|}
\hline \multirow{2}{*}{ Iterations } & \multicolumn{3}{|c|}{ ISNR } \\
\cline { 2 - 4 } & TV norm & $\mathbf{l}_{\mathbf{1}}$ norm & $\mathbf{l}_{\mathbf{0}}$ norm \\
\hline 500 & 6.275 & 6.285 & 6.285 \\
\hline 1000 & 6.690 & 6.688 & 6.688 \\
\hline 1500 & 6.985 & 6.955 & 6.955 \\
\hline 2000 & 7.210 & 7.174 & 7.210 \\
\hline 2500 & 7.398 & 7.125 & 7.367 \\
\hline 3000 & 7.557 & 7.149 & 7.530 \\
\hline 3500 & 7.655 & 7.141 & 7.648 \\
\hline 4000 & 7.786 & 7.137 & 7.781 \\
\hline 4500 & 7.865 & 7.157 & 7.879 \\
\hline 5000 & 7.993 & 7.163 & 7.974 \\
\hline
\end{tabular}


A Study on Regularization Functions and Regulation Parameters in Image Restoration

Table 2 ITERATIONS vs. Objective function using ISTA Blur size 9x9

\begin{tabular}{|c|c|c|c|}
\hline \multirow{2}{*}{ Iterations } & \multicolumn{3}{|c|}{ Objective function } \\
\cline { 2 - 4 } & $\mathbf{T V}$ norm $\left.\mathbf{( 1 0}^{\mathbf{3}}\right)$ & $\mathbf{l}_{\mathbf{1}}$ norm(10 \\
$\mathbf{4}$ & $\mathbf{I}_{\mathbf{0}} \mathbf{n o r m}\left(\mathbf{1 0}^{\mathbf{3}}\right)$ \\
\hline 500 & 9.54921 & 2.81700 & 5.42570 \\
\hline 1000 & 9.23162 & 2.80714 & 5.18368 \\
\hline 1500 & 9.09400 & 2.79831 & 6.95500 \\
\hline 2000 & 9.05012 & 2.80361 & 4.98930 \\
\hline 2500 & 8.91964 & 2.79718 & 4.88313 \\
\hline 3000 & 8.92602 & 2.80016 & 4.89099 \\
\hline 3500 & 8.92025 & 2.80091 & 4.85595 \\
\hline 4000 & 8.92264 & 2.79913 & 4.83579 \\
\hline 4500 & 8.80493 & 2.80014 & 4.81461 \\
\hline 5000 & 8.84212 & 2.80633 & 4.84594 \\
\hline
\end{tabular}

Table 3 ITERATIONS Vs ISNR and Objective function using IISTA Blur size 9x9

\begin{tabular}{|c|c|c|c|c|}
\hline \multirow{2}{*}{ Iterations } & \multicolumn{2}{|c|}{ ISNR } & \multicolumn{2}{c|}{ Objective function } \\
\cline { 2 - 5 } & TV norm & $\mathbf{l}_{\mathbf{0}}$ norm & $\begin{array}{c}\text { TV norm } \\
\mathbf{( 1 0}^{\mathbf{3}} \mathbf{~}\end{array}$ & $\mathbf{b}_{\mathbf{~ n o r m}\left(\mathbf{1 0}^{\mathbf{3}} \mathbf{)}\right.}$ \\
\hline 50 & 6.613 & 6.160 & 9.70883 & 5.53750 \\
\hline 100 & 6.951 & 6.972 & 9.14136 & 5.02918 \\
\hline 150 & 7.612 & 7.622 & 9.01817 & 4.81195 \\
\hline 200 & 8.077 & 8.127 & 8.81832 & 4.78740 \\
\hline 250 & 8.452 & 8.410 & 8.81019 & 4.79088 \\
\hline 300 & 8.660 & 8.612 & 8.81834 & 4.80032 \\
\hline 350 & 8.452 & 8.682 & 8.79188 & 4.72642 \\
\hline 400 & 8.750 & 8.801 & 8.78827 & 4.76248 \\
\hline 450 & 8.719 & 8.788 & 8.80850 & 4.79617 \\
\hline 500 & 8.736 & 8.718 & 8.79433 & 4.78165 \\
\hline
\end{tabular}

Table 4 ITERATIONS vs. ISNR using IISTA Blur size4x4

\begin{tabular}{|c|c|c|c|}
\hline \multirow{2}{*}{ Iterations } & \multicolumn{3}{|c|}{ ISNR } \\
\cline { 2 - 4 } & TV norm & $\mathbf{l}_{\mathbf{1}}$ norm & $\mathbf{l}_{\mathbf{0}}$ norm \\
\hline 50 & 6.649 & 6.431 & 6.610 \\
\hline 60 & 6.841 & 6.523 & 6.710 \\
\hline 70 & 7.037 & 6.525 & 6.753 \\
\hline 80 & 7.130 & 6.523 & 6.852 \\
\hline 90 & 7.322 & 6.544 & 6.880 \\
\hline 100 & 7.482 & 6.549 & 6.903 \\
\hline 110 & 7.560 & 6.578 & 6.891 \\
\hline 120 & 7.651 & 6.554 & 6.842 \\
\hline 130 & 7.764 & 6.535 & 6.891 \\
\hline 140 & 7.823 & 6.521 & 6.909 \\
\hline 150 & 7.918 & 6.606 & 6.827 \\
\hline
\end{tabular}


Table 5 Regularization parameter $\boldsymbol{\tau}$ vs. ISNR using ISTA Number of Iterations $=50$

\begin{tabular}{|c|c|c|c|}
\hline \multirow{2}{*}{$\tau$} & \multicolumn{3}{|c|}{ ISNR } \\
\cline { 2 - 4 } & TV norm & $\mathbf{l}_{\mathbf{1}}$ norm & $\mathbf{I}_{\mathbf{0}}$ norm \\
\hline $10^{-1}$ & 6.427 & 5.916 & 5.920 \\
\hline $10^{-2}$ & 6.002 & 6.000 & 6.002 \\
\hline $10^{-3}$ & 5.941 & 5.879 & 5.930 \\
\hline $10^{-4}$ & 5.902 & 5.923 & 5.936 \\
\hline $10^{-5}$ & 5.894 & 5.898 & 5.895 \\
\hline $10^{-6}$ & 5.901 & 5.895 & 5.932 \\
\hline $10^{-7}$ & 5.921 & 5.894 & 5.882 \\
\hline $10^{-8}$ & 5.914 & 5.902 & 5.914 \\
\hline $10^{-9}$ & 5.899 & 5.921 & 5.916 \\
\hline $10^{-10}$ & 5.905 & 5.914 & 5.924 \\
\hline $10^{-20}$ & 5.850 & 5.899 & 5.898 \\
\hline $10^{-30}$ & 5.898 & 5.905 & 5.891 \\
\hline $10^{-38}$ & 5.89 & 5.899 & 5.912 \\
\hline
\end{tabular}

Table 6 Regularization parameter $\boldsymbol{\tau}$ vs. Objective function using ISTA Number of Iterations $=50$

\begin{tabular}{|c|c|c|c|}
\hline \multirow{2}{*}{$\tau$} & \multicolumn{3}{|c|}{ Objective function } \\
\cline { 2 - 4 } & TV norm $\left.\mathbf{( 1 0}^{4}\right)$ & $\mathbf{l}_{\mathbf{1}}$ norm $\left.\mathbf{( 1 0}^{4}\right)$ & $\mathbf{l}_{\mathbf{0}}$ norm $\left(\mathbf{1 0}^{4}\right)$ \\
\hline $10^{-1}$ & 6.9238 & 28.1120 & 1.1537 \\
\hline $10^{-2}$ & 1.3130 & 3.2429 & 0.5330 \\
\hline $10^{-3}$ & 0.5531 & 0.7370 & 0.4730 \\
\hline $10^{-4}$ & 0.4734 & 0.4958 & 0.4620 \\
\hline $10^{-5}$ & 0.4570 & 0.4635 & 0.4606 \\
\hline $10^{-6}$ & 0.4600 & 0.4560 & 0.4597 \\
\hline $10^{-7}$ & 0.4576 & 0.4561 & 0.4589 \\
\hline $10^{-8}$ & 0.4600 & 0.4623 & 0.4644 \\
\hline $10^{-9}$ & 0.4614 & 0.4576 & 0.4579 \\
\hline $10^{-10}$ & 0.4630 & 0.4600 & 0.4629 \\
\hline $10^{-20}$ & 0.4584 & 0.4614 & 0.4641 \\
\hline $10^{-30}$ & 0.4584 & 0.4629 & 0.4617 \\
\hline $10^{-38}$ & 0.4594 & 0.4649 & 0.4556 \\
\hline
\end{tabular}

Table 7 Regularization parameter $\boldsymbol{\tau}$ vs. ISNR using IISTA Number of iterations $=50$

\begin{tabular}{|c|c|c|c|}
\hline \multirow{2}{*}{$\tau$} & \multicolumn{3}{|c|}{ ISNR } \\
\cline { 2 - 4 } & TV norm & $\mathbf{l}_{\mathbf{1}}$ norm & $\mathbf{I}_{\mathbf{0}}$ norm \\
\hline $10^{-1}$ & 6.780 & 5.959 & 5.974 \\
\hline $10^{-2}$ & 6.544 & 6.543 & 6.554 \\
\hline $10^{-3}$ & 5.928 & 5.913 & 5.942 \\
\hline $10^{-4}$ & 5.890 & 5.935 & 5.910 \\
\hline $10^{-5}$ & 5.934 & 5.909 & 5.898 \\
\hline $10^{-6}$ & 5.931 & 5.917 & 5.994 \\
\hline $10^{-7}$ & 5.906 & 5.945 & 5.915 \\
\hline $10^{-8}$ & 5.924 & 5.892 & 5.917 \\
\hline $10^{-9}$ & 5.860 & 5.926 & 5.933 \\
\hline $10^{-10}$ & 5.907 & 5.928 & 5.908 \\
\hline $10^{-20}$ & 5.920 & 5.938 & 5.910 \\
\hline $10^{-30}$ & 5.916 & 5.890 & 5.958 \\
\hline $10^{-38}$ & 5.934 & 5.906 & 5.930 \\
\hline
\end{tabular}


A Study on Regularization Functions and Regulation Parameters in Image Restoration

Table 8 Regularization parameter $\boldsymbol{\tau}$ vs. Objective function using IISTA Number of iteration $=50$

\begin{tabular}{|c|c|c|c|}
\hline \multirow{2}{*}{$\tau$} & \multicolumn{3}{|c|}{ Objective function } \\
\cline { 2 - 4 } & TV norm $\left(\mathbf{1 0}^{4}\right)$ & $\mathbf{l}_{\mathbf{1}}$ norm $\left(\mathbf{1 0}^{\mathbf{4}}\right)$ & $\mathbf{L}_{\mathbf{0}}$ norm $\left.\mathbf{( 1 0}^{\mathbf{4}}\right)$ \\
\hline $10^{-1}$ & 5.8518 & 28.1210 & 1.1564 \\
\hline $10^{-2}$ & 1.2533 & 3.2316 & 0.5159 \\
\hline $10^{-3}$ & 0.5021 & 0.6792 & 0.4112 \\
\hline $10^{-4}$ & 0.4031 & 0.4319 & 0.3946 \\
\hline $10^{-5}$ & 0.3986 & 0.3999 & 0.4002 \\
\hline $10^{-6}$ & 0.3994 & 0.3969 & 0.3963 \\
\hline $10^{-7}$ & 0.3995 & 0.3956 & 0.3981 \\
\hline $10^{-8}$ & 0.3969 & 0.3952 & 0.4023 \\
\hline $10^{-9}$ & 0.3952 & 0.4002 & 0.3961 \\
\hline $10^{-10}$ & 0.3925 & 0.3938 & 0.3965 \\
\hline $10^{-20}$ & 0.3957 & 0.3992 & 0.3959 \\
\hline $10^{-30}$ & 0.3991 & 0.3967 & 0.3924 \\
\hline $10^{-38}$ & 0.3976 & 0.3995 & 0.4001 \\
\hline
\end{tabular}

Figure 1 and Figure 2 shows restoration using different norms of ISTA and IISTA algorithms.

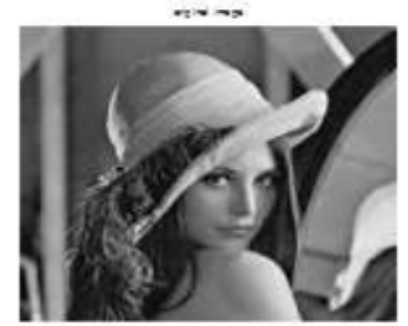

(A)

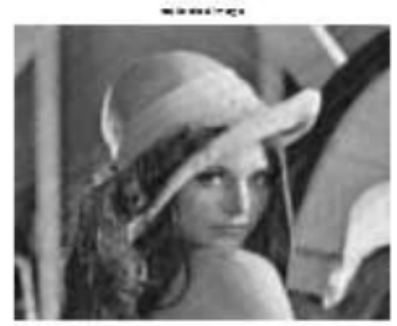

(A)

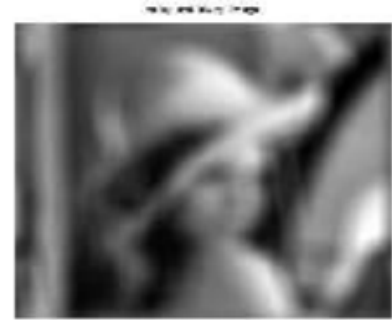

(B)

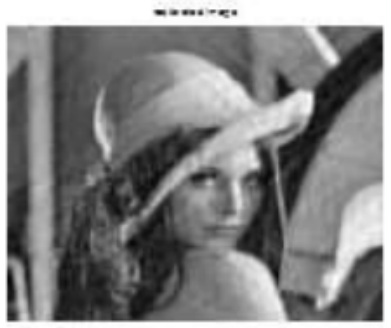

(B)

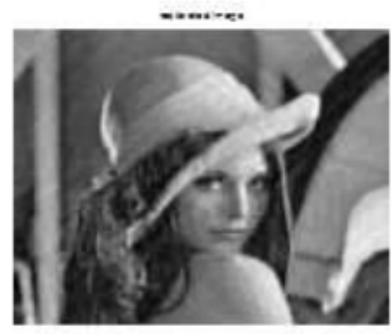

(E)

Figure 1 A) Original image B) noisy and blur image Blur size 9x9 Restorated using ISTA C) TV norm D) 11 norm and E) 10 norm Number of iterations is 5000 . 


\section{A.K. Kumaresh}

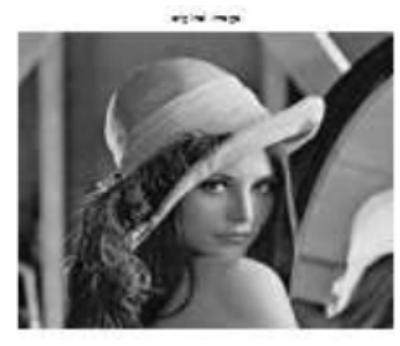

(A)

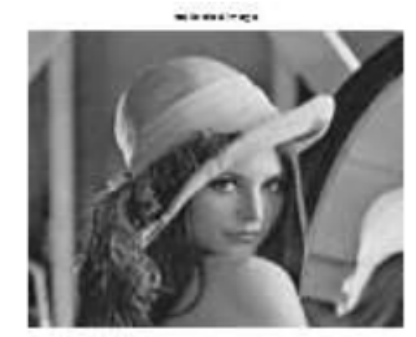

(A)

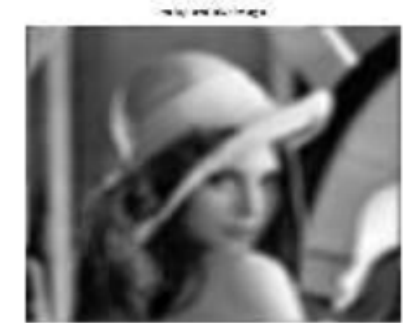

(B)

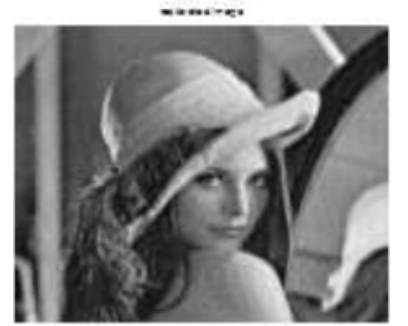

(B)

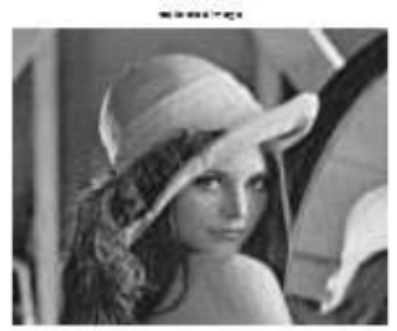

(E)

Figure 2 A) Original image B) noisy and blur image blur size 4x4 Restorated using IISTA C) TV norm D) $l_{1}$ norm and E) $l_{0}$ Number of Iterations is 150

\section{CONCLUSION}

The observations shows that under blur image of size $9 \times 9$ and $4 \times 4$, TV norm shows better ISNR than $l_{0}$ norm and $l_{1}$ norm for both ISTA and IISTA. In the case of $1_{1}$ norm using 9x9 blur image size shows recovery is not possible after a certain number of iterations. The study of regularization parameter shows better performance for $l_{0}$ norm than TV norm and $l_{1}$ norm. It is observed that when the value of $\boldsymbol{\tau}$ decreases the performance of algorithms is also decreases. The future study may be continued for the role of different types of noise.

\section{REFERENCES}

[1] "Digital Image Processing", by R.C.Gonzalez and R.E.Woods, Addision Wesley An imprint of Pearson Education

[2] "Fundamentals of Digital Image Processing", by A.K .Jain

[3] Digital Image Processing Using MATLAB by R.C.Gonzalez R.E.Woods and S.L.Eddins Pearson Education 
A Study on Regularization Functions and Regulation Parameters in Image Restoration

[4] I.Daubechies, M.D.Friese and C.D.Mol, "An Iterative thresholding algorithm for linear inverse problems with sparsity constraint", Commu.Pure Appl. Math. Vol 57, pp 14131457,2004

[5] M.Elad P.Milanfar and R.Rubinstein, "An Analysis Versus Synthesis in signal priors", Inv. Probl. Vol 23, pp 947-968, 2007

[6] T.Chan S. Esedoglu F Park and A Yip, "Recent developments in total variation image Restoration", in Handbook of mathematical models in computer vision N.Paragios, Y.Chen and O.Faugeras, Eds New York: Springer -Verlag, 2005

[7] L.Rudin ,S,Osher and E.Fatemi, "Non-linear total variation based noise removal algorithms', Physica D vol 60,pp 259-268,1992

[8] D.Mumford and J.shah, "optimal approximation by piecewise smooth functions and associated variational problems", Commun. Pure Appl. Math 42 (1982) pp 577-685

[9] "Fast Image Recovery using variable splitting and constrained optimization ", M.V.Afonso, J.M.Bioucas-Dias, A.T. Figueiredo. IEEE Transactions on Image Processing.vol 19, No 9, Sep 2010

[10] A paper entitled "Image Restoration by Improved Iterative Shrinkage Thresholding Algorithm" ,A.K.Kumaresh, International Journal of Computer Engineering \& Technology, volume 10,Issue 2,March-April,2019,pp 91-98.Journal Impact Factor(2019):10.5167 (Calculated by GISI) ISSN Print:0976-6367 and ISSN Online:09766375 UGC Reference Number:45405

[11] A paper entitled " Image Restoration using IIST Algorithm" is presented on "International Conference on Intelligent Computing Applications" at Department of Computer Applications, Bharathiar University, Coimbatore TamilNadu India Under the Sponsorship of UGC and DST on March 6-7 2014.The paper affiliation published by IEEE-CPS and the corresponding ISBN 978-1-4799-3966-4. pp 258-262 The copy right of the paper is owned by IEEE.

[12] M Betero and P Boccacci, “ Introduction to Inverse Problems in imaging” Bristol U.K. IOP 1998.

[13] A.Chambolle ,"An Algorithm for total variation minimization and applications", J.Math Imag. Vis., vol 20 pp 89-97, 2004

[14] D.Donoho and I.Johnson,"Adapating to unknown smoothness via wavelet shrinkage", J.Amer Statist Assoc vol 90 no 432 pp 1200-1224, 1995.

[15] D.Donoho,'Denoising by soft thresholding”, IEEE Trans Inf. Theory vol 41 no 3 pp 613627 May 1995. 